PII S0735-1933(97)00055-9

\title{
THE EXTINCTION LIMITS AND NEAR-LIMITS BEHAVIORS OF PREMIXED ETHANOL/AIR FLAME
}

\author{
Ching-Hua Wang, Gwo-Jiun Ueng and Jyh-Jer Jehng \\ Department of Mechanical Engineering \\ National Taiwan University \\ Taipei, Taiwan, Republic of China
}

(Communicated by J.P. Hartnett and W.J. Minkowycz)

\begin{abstract}
Experimental studies on the extinction limits and near-limits flame behaviors of premixed ethanol/air flames in a stagnation point flow were made by using an axisymmetric counter flow, twin-flame configuration, with adjustable nozzles separation distance. The extinction limits were obtained under various conditions included equivalence ratios, flame stretch rates, inlet mixture temperatures, with and without heat loss; and the flammability of ethanol was then obtained by extrapolating the extinction limits to a stretchless condition. Finally, various nearlimits flame extinction phenomena were discussed. (1) 1997 Elsevier Science Ltd
\end{abstract}

\section{Introduction}

In the past two decades extensive progress in the oxidation kinetics of alcoholic fuels has been made. Such a progress was facilitated with two major advances which allowed close coupling between experimental and computational investigations. The first is the computational simulation and comparison of combustion phenomena and parameters with detailed chemistry $[1,2]$. Through such a comparison the adequacy and weaknesses of a given reaction scheme can be frequently identified by using, for example, sensitivity and reaction path analyses. The various 
experimental information used for such a comparison includes the ignition delays, the laminar burning velocities, the extinction stretch rates, and the scalar structure of the flames. The second factor is the recognition $[3,4]$ that previous experimentally determined values of laminar burning velocities and extinction limits were frequently, and extensively, complicated by stretch effects such that significant uncertainties arose when they were used to "validate" or "partially validate" a kinetic mechanism. Law and $\mathrm{Wu}$ [3] and Law [4] subsequently proposed the counter flow twinflame technique to systematically subtract out these stretch effects by first determining appropriate reference burning velocities and extinction limits of flames with various stretch rates, and then linearly extrapolating these reference burning velocities and extinction limits to zero stretch rate to yield the stretchless laminar burning velocity and flammability limit. This technique has since been refined to allow for nonlinear extrapolation $[5,6]$ when the nozzle separation distance is not sufficiently large and/or the Karlovitz number not sufficiently small [7]. Meanwhile, the general concept of including stretch effects in processing experimental data has also been extended to spherical flames $[8,9,10]$

Although many advances in flame kinetic have been made on alcoholic fuels, the qualified experimental data on those fuels are relatively limited. Thus, the present investigation provides experimental data of accuracy on the extinction stretch rates of ethanol/air flames, using the counter flow technique, with the anticipation that these data will be useful for further studies on their oxidation. We also investigate the effects of downstream heat loss on the flame extinction, and provide quantitative information on the magnitudes of such influences. The experimental extinction stretch rates determined herein are also expected to be of value to the study of turbulent flame and engine combustion, because the curvature associated with those flames lead easier extinction, thereby producing more pollutants if special care is not given.

The experimental methodology and the results presented herein presented in the next two sections

\section{Experimental Methodology}

The experimental apparatus (Fig. 1) consists of a 1-D LDA system, a 3-axis traversing stage, an axisymmetric counter flow burner, fuel and air supplies, particle seeding units, and heating elements with voltage regulators. The LDA system is composed of a $25 \mathrm{mw} \mathrm{He}-\mathrm{Ne}$ Laser with a modular beam splitter and Dantec Type 55N11 Frequency Shifter, which is then placed on the 3axis traversing stage with a resolution of $0.025 \mathrm{~mm}$. The forward scattered light signals from the 
$0.1 \sim 1 \mu \mathrm{m} \mathrm{MgO}$ seeding particles are accumulated through a photomutiplier; the average scattered light frequency is computed using a Dantec $58 \mathrm{~N} 40$ signal processor and Dantec FLOware software package to compute the average particle velocity. The axisymmetric counter flow burner consists of two identical opposed nozzles, with an area contraction ratio of $9: 1$, an exit diameter of $18 \mathrm{~mm}$, nitrogen co-flow, and the separation distance between the two nozzles is adjustable

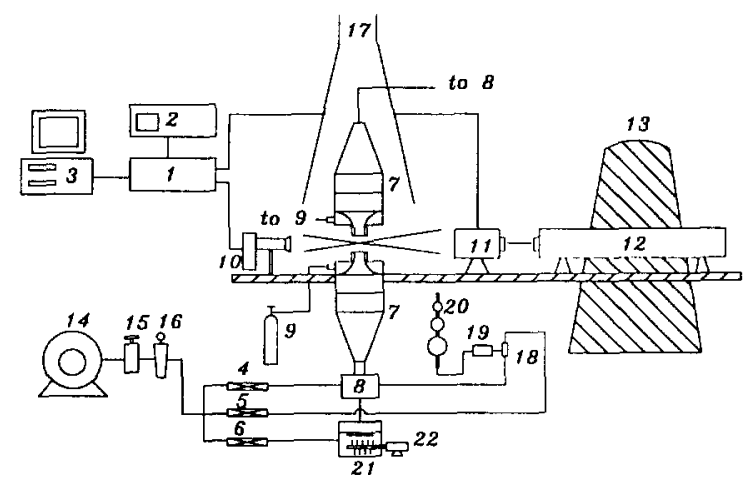

$\begin{array}{llll}\text { 1. mean vel. computer } & \text { 7. burner } & \text { 13. traversing stage } & \text { 19. fuel supply } 1 \\ \text { 2. frequency shifter } & \text { 8. mixing chamber } & \text { 14. air compressor } & \text { 20. fuel supply } 2 \\ \text { 3. monitor } & \text { 9. nitrogen } & \text { 15. air filter } & \text { 21. particle feeder } \\ \text { 4. main air meter } & \text { 10. photo multiplier } & \text { 16. regulator } & \text { 22. motor } \\ \text { 5. atomizer air meter } & \text { 11. beam splitter } & \text { 17. ventilation } & \\ \text { 6. seeding air meter } & \text { 12. laser } & \text { 18. atomizer }\end{array}$

FIG. 1

The schematic diagram of the experimental apparatus

The LDA system was first calibrated by a motor driven rotational disc in the velocity range from 0.4 to $2 \mathrm{~m} / \mathrm{sec}$ and the accuracy was found to be $\pm 0.3 \%$. The cold air radial velocity profile at the nozzle exit were tested and the distribution of them at different air flow rates were found to be uniform at most central regions and rapidly changed only close to the free boundary, thereby demonstrating that the design of the nozzle is satisfactory.

Technical grade liquid ethanol with a purity exceeding $99.5 \%$ were steadily supplied by two independent and identical d.c. micro-gear pumps; by the aid of venturas, liquid ethanol formed spray which then vaporized either within air stream or on the hot surface of copper tubing. The rate of ethanol supply was linearly proportional to the voltage supplied to the pumps. The temperature of the hot surface of copper tubing was carefully controlled as not to exceed $200 \mathrm{C}$ to prevent thermal pyrolysis of ethanol. Electric heating coils and thermal insulators were placed 
around all mixture supply lines and burners to prevent ethanol vapor from condensing in the flow circuit

A small amount of air went through the particle seeding unit and brought a sufficient amount of $\mathrm{MgO}$ particles to the mixture as the velocity reference for LDA. Since the sizes of seeding particles were small which followed the mixture flow without much lag. Thus, the velocity measured by LDA can be considered as the flow velocity, particularly in the upstream of flame [11]. Additional air was then supplied to have proper overall equivalence ratio of the mixture and'or stretch rate of the flow.

The mixture temperature was controlled by the supplied voltages to the heating coils and measured at the exit plane of nozzle by a J-type thermocouple. The mixture composition was calculated from the individual supply rates of ethanol and air, and it was also checked by Shimadzu GC-14A Gas Chromatography at the exit plane of nozzle.

When the premixed combustible mixture is ignited, the twin stationary flame surfaces adjust to their proper positions. As the mixture passes the flame surfaces, it reacts and the temperature increases from original nozzle exit temperature to flame temperature within a short distance; while flow velocity after the flame suddenly increases due to the effect of thermal expansion. Thus, flow fields that with and without flame are complete difference. Flows that have the same overall flow rate but different equivalence ratios have different flame positions. All those flames then lead to different flame stretch rates. Therefore, obtaing the velocity profiles in each cases to derive a complete set of the flame stretch ratës is extremely difficult.

Two types of experiments could be performed allowing for various degrees of downstream heat transfer. In an axisymmetrical, twin-flame configuration, properties of the combustibles issued from the two nozzles could be identical. Thus, upon ignition, two identical flames were produced on the two sides of the stagnation surface. Due to symmetry, the extent of heat loss downstream of the flame can be considered to be minimal and the flames as well as the extinction are therefore approximately adiabatic. In the second arrangement, the upper combustible mixture was replaced by room-temperature nitrogen such that only one flame was produced. Furthermore, since the downstream boundary temperature for this flame is the room temperature, this nitrogen-opposed flame not only is sub-adiabatic through downstream heat loss, but the extent of downstream heat loss can also be considered to be maximum. The flame extinction limits with and without downstream heat loss were then investigated under these two different 
arrangements of upper mixture's conditions.

By carefully adjusting the air, fuel and nitrogen co-flow rates as well as the heating power, stable stationary twin flames were obtained. The flow velocity was then measured along the center axis from $3 \sim 4 \mathrm{~mm}$ upstream of the flame to a location slightly downstream of the velocity minimum. Figure 2 displays a typical velocity profile of the lower burner for the adiabatic, twinflame configuration. This velocity profile then allowed velocity gradient, $d V / d x$, to be determinated, which is defined as the negative of flame stretch rate, $K$. In this investigation, the flame stretch rates were varied from 120 to $410 \mathrm{l} / \mathrm{sec}$, while the initial mixture temperatures were varied from 50 to $150 \mathrm{C}$.

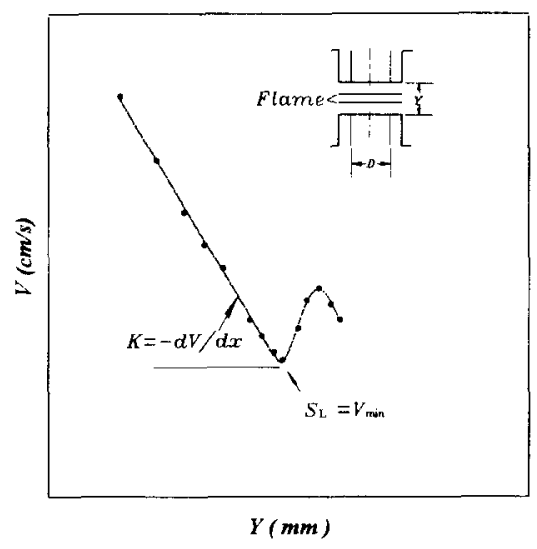

FIG. 2

Typical velocity profile along the center axis of twin-flame

For a given mixture composition and preheated temperature, the extinction flame stretch rate was determined by first put the separation distance of two nozzles is as far as possible, and then gradually close the distance to increase velocity gradient, then a relative position is reached for which extinction would occur by slightly decreasing the distance. We then used LDA to measure the velocity profile at that position; thus the flame stretch rate which cause the flame extinction at the given mixture equivalence ratio and temperature could be determined. By varying mixture compositions and heating powers, and repeating the same procedures, the extinction flame stretch rates at different mixture equivalence ratios and temperatures were then determined.

Collecting all the results at the same mixture temperature, we then have the plot of the 
extinction equivalence ratios versus the corresponding extinction flame stretch rates. By further extrapolating the results to a zero stretch condition, the stretchless extinction equivalence ratio at that preheated mixture temperature was then obtained, which we define it as the flammability limit of mixture at that temperature.

Near-limits flame temperature distributions along the center axis were measured by a B-type thermocouple (Pt $6 \% \mathrm{Rh} / \mathrm{Pt} 30 \% \mathrm{Rh}$ ) with wire size of $0.2 \mathrm{~mm}$ coated with $\mathrm{S}_{1} \mathrm{O}_{2}$. The relative flame positions as well as the flames separation distances were also obtained in the mean time to characterize the flame extinction behaviors.

\section{Results and Discussion}

\section{The Effect of Flame Stretch on the Flame Extinction Limits}

Figure 3 presents the results for the lean flame extinction equivalence ratios $(\phi)$ versus the extinction flame stretch rates of ethanol/air mixture at mixture temperatures of 50,100 and $150 \mathrm{C}$.

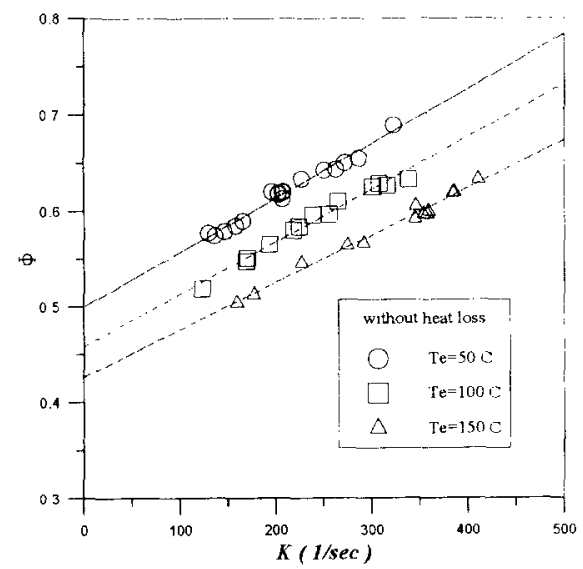

FIG. 3

The flame extinction equivalence ratios $(\varphi)$ vs. the flame stretch rates for the lean mixtures at different temperatures

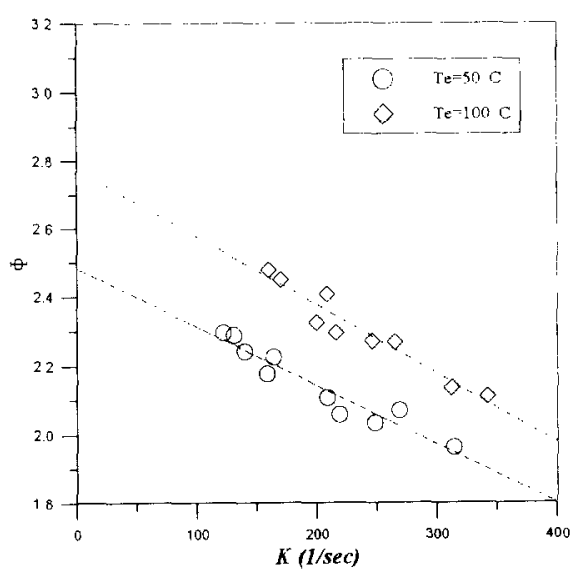

FIG. 4

The flame extinction equivalence ratios $(\phi)$ vs. the flame stretch rates for the rich mixtures at different temperatures

The meaning of each data point is - the existing flames will be extinguished either by increasing the flame stretch rate at a fixed equivalence ratio of the mixture, or by decreasing the equivalence ratio at fixed flame stretch rate. The line connecting the data points with the same mixture temperature can then be considered as the lean flame extinction limit of the mixture at that given 
temperature. The conditions of mixture which on the right or bottom of the line have stable flames.

Figure 4 depicts the results for the rich flame extinction equivalence ratios versus the extinction flame stretch rates of ethanol/air mixture at mixture temperatures of 50 and $100 \mathrm{C}$. At the rich flame extinction limits, the stable flames can be extinguished by increasing the flame stretch rate or equivalence ratio of the mixture at a given mixture temperature. The conditions of mixture which on the left or bottom parts of the connecting lined have stable flames.

Damkoler number $(D a)$ of mixture in $\mathrm{S}$ curve is defined as the characteristic resident time divided by the characteristic reaction time [12]. And if the resident time is longer than the time needed for the reaction to be complete. Then, the flame can be stabilized; adversely, the flame will be extinguished. Thus, one may define an extinction Damkoler number, $D a_{e x}$, as an extinction criteria. For which if $D a$ of the mixture is greater than $D a_{e x}$ there is a stable flame; and adversely, the flame will be extinguished.

The extinction results can thus be accounted for quite well from the Damkoler number point of view. For a mixture, a $D a_{e x}$ should exist, by decreasing the resident time (increasing the flame stretch rate or overall flow rate) or increasing the reaction time (decreasing the reactivity or decreasing (increasing) the equivalence ratio in lean (rich) mixture) will lead $D a$ of the mixture decrease. Once $D a$ of the mixture is less than $D a_{e x}$, the flame will then be extinguished. Figures 3 and 4 depict clear trends both in the lean and the rich conditions.

The rate effects of flame stretch rate on the lean and rich extinction equivalence ratio are $\sim 5$ $\times 10^{-4}\left(3.5 \times 10^{-3} \mathrm{vol} . \%\right)$ and $20 \times 10^{-4}\left(10 \times 10^{-3} \mathrm{vol} \%\right)$ - sec for ethanol/air mixtures, respectively. Obviously, the effects of flame stretch rate on the lean extinction limits is weaker than that on the rich extinction limits

The Effect of Preheated Mixture Temperature on the Flame Extinction Limits

Figures 3 and 4 present the effects of mixture temperature on the flame extinction limits were also presented. As those figures reveal, the lean (rich) extinction equivalence ratios at the same flame stretch rate decreased (increased) as the mixture temperature increased.

From a Damköler number perspective, the higher the preheated mixture temperature implies a higher flame temperature, thereby leading to an increasing reactivity or $D a$ of the given mixture. 
Thus, the flame is more difficult to be extinguished. In other words, a stable flame will be extinguished by either through further decreasing the resident time or increasing the chemical reaction time at an elevated mixture temperature.

Figure 5 further present the results of the lean extinction limits with downstream heat loss by replacing the upper combustible mixture with room-temperature nitrogen. On the figure, the results of twin flames were also included for the comparison, revealing that downstream heat loss played a prominent effect on the extinction limits. In fact, the practical extinction limits of a mixture should lie between these two sets of values, since the data points on figure represent the minimal and the maximum heat loss condition, respectively.

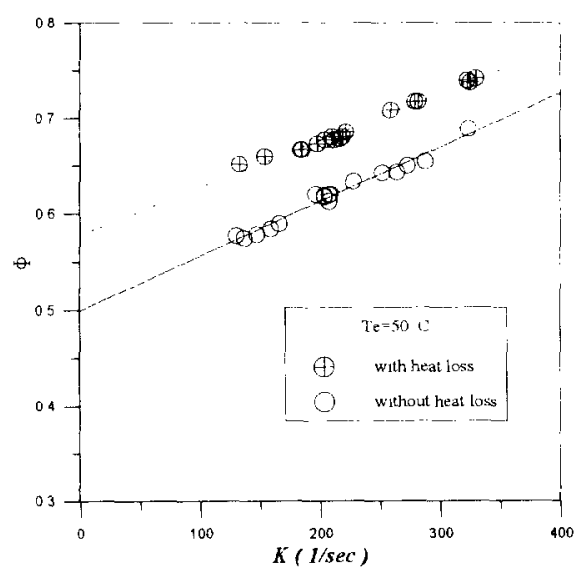

FIG. 5

The flame extinction equivalence ratios vs. the flame stretch rates for the lean mixtures, with and without heat loss

\section{The Decision of the Flammability Limits}

The fundamental definition of flammability limits is a flame that can not self propagate within a given fuel/air mixture. Conventionally, they were all determined by the flame tube or constant volume bomb approaches [13]. Law et al. [14] initially extrapolated their results on the extinction equivalence ratios vs. the flame stretch rates to a zero stretch condition of methane/air and propane/air mixtures, finding those results to be close to the flammability limits obtained by the flame tube approach. Although the approach is much more tedious, it is more meaningful and has demonstrated the effect of a flame stretch rate. We then applied the same approach and obtained the lean and rich flammability for ethanol/air mixture at different mixture temperatures. 
For ethanol/air mixture, the extrapolating results of the lean flammability limits were 0.50 , 0.46 and $0.43(3.38,3.12$ and $2.92 \mathrm{vol} \%)$ at temperatures of 50,100 and $150 \mathrm{C}$, respectively; and the rich flammability limits were 2.48 and 2.78 (14.8 and $16.3 \mathrm{vol} \%)$ at temperatures of 50 and $100 \mathrm{C}$. The effects of mixture temperature on the lean and rich flammability limits are $\sim 7 \mathrm{x}$ $10^{-4}\left(4.6 \times 10^{-3} \mathrm{vol} . \%\right)$ and $60 \times 10^{-4}\left(30 \times 10^{-3} \mathrm{vol} . \%\right) / \mathrm{C}$, respectively.

The lean and rich flammability limits of ethanol/air mixtures from Glassman [15], obtained by flame tube approach, were $0.5(3.38 \mathrm{vol} \%)$ at $25 \mathrm{C}$ and $2.9(16.9 \mathrm{vol} . \%)$ at $60 \mathrm{C}$, respectively. And if we linearly related our results to temperatures of $25 \mathrm{C}$ (lean) and $60 \mathrm{C}$ (rich), we would have the values of $\sim 0.51(3.45 \mathrm{vol} \%)$ and $2.54(15.1 \mathrm{vol} \%)$, respectively. Some discrepancies arise between the results for the rich flammability, which will be discussed in the later sections

\section{The Extinction Behaviors near the Extinction Limits}

During experimental study on the extinction limits, at the fixed mixture's equivalence ratio and then decreased the separation distance between the two nozzles, one could observe that the twin flames will toward to each other and finally get extinguished.

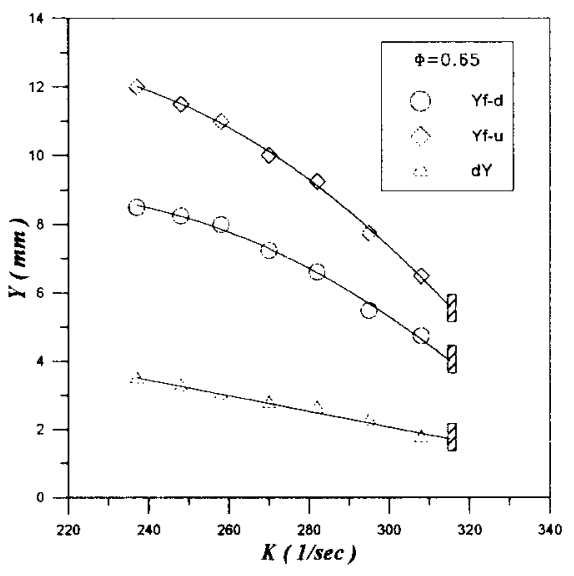

FIG. 6

Flames relative positions and their separation distance at different flame stretch rates for mixture equivalence ratio of 0.65

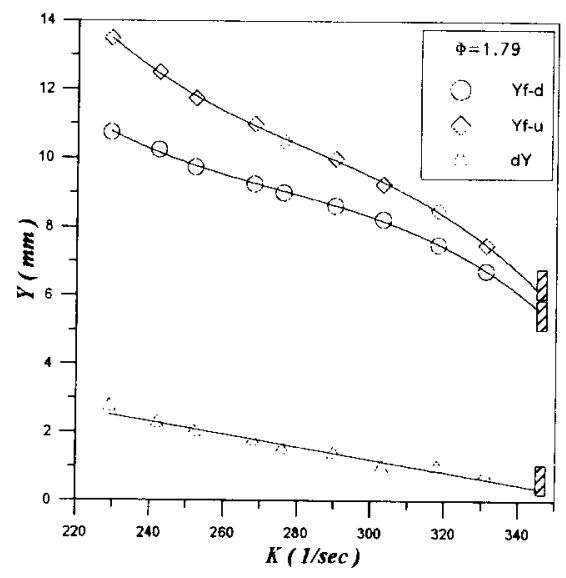

FIG. 7

Flame relative positions and their separation distances at different flame stretch rates for mixture equivalence ratio of 1.79

However, at the point that the flames are extinguished, the phenomena are quite difference among the extinguished flames. Figures 6 and 7 show some typical results of the relative flame positions and their separation distances for ethanol/air mixtures at mixture temperature of $50 \mathrm{C}$ 
with equivalence ratios of 0.65 and 1.79 at different flame stretch rates, respectively; where $y=0$ $\mathrm{mm}$ is the position of the exit plane of lower nozzle. As can be seen from the figures, for the lean ethanol flames, the two opposed flames were extinguished with a finite separation distance. However, for the rich flames, the two flames were almost merged at extinction

The behaviors can then be accounted for from the perspective of non-unity Lewis number $\left(\mathrm{Le}=\alpha / D_{i}\right)$ effect and/or preferential diffusion $\left(D_{i} / D_{j}\right)$ effect of the mixture as the flames suffer a stretch (where $\alpha$ is the thermal diffusivity, $D_{i}$ denotes the mass diffusivity of the deficient reactant and $D_{j}$ represents the mass diffusivity of the excess reactant in mixture), which had been stated quite clearly in references $[14,16]$.

According to Sato [16], experimental studies on the counter flow flames of methane/air and propane/air mixtures were investigated, finding that, when the deficient species in mixture is the less mobile reactant (such as $\mathrm{C}_{3} \mathrm{H}_{8}$ in the lean $\mathrm{C}_{3} \mathrm{H}_{8}$ /air mixture or $\mathrm{O}_{2}$ in the rich $\mathrm{CH}_{4} /$ air mixture), Le $>1$ for the mixtures; as the flames suffer a positive stretch, heat loss exceeds mass gain, increasing in flame stretch rate decreases the reaction intensity and ends with flame extinction. At extinction, the reaction is complete and the flames are distant from the stagnation surface. On the other hands, when the deficient species is the more mobile reactant (such as $\mathrm{CH}_{4}$ in the lean $\mathrm{CH}_{4}$ /air mixture or $\mathrm{O}_{2}$ in the rich $\mathrm{C}_{3} \mathrm{H}_{8}$ /air mixture), Le $<1$ for the mixtures; heat loss less mass gain, the reaction intensity continuous increases with the increasing in flame stretch rate until the two flames contact with each other. Further increasing the flame stretch rate causes an incomplete reaction due to a decrease in the resident time which subsequently causes flames be extinguished.

Preferential diffusion can also interpret such phenomena. In mixtures of methane/air, methane is the more diffusive reactant. Thus in the lean condition, the reactant concentration in reaction zone becomes more stoichiometric and burns more intense. Thus, extinction only occurs when the flames approach to each other and the reaction is incomplete. On the other hand, in the rich condition, the reactant concentration becomes more off-stoichiometric, the reaction intensity is weakened even when the reaction is complete; extinction occurs with a finite separation distance. For propane/air mixtures, since propane is the less diffusive reactant, the phenomena is thus adverse to that of methane/air mixtures - extinction occurs with a finite separation distance of the two flames in the lean condition, and the two flames are getting very close in the rich condition. 
For ethanol/air flames, since the molecular weight of ethanol is heavier than that of oxygen, the effective Lewis number are 1.731 and 0.972 at mixture equivalence ratios of 0.65 and 1.79 , respectively, which were calculated by the Sandia computer code [17]. Thus, both the effects of non-unity Lewis number and preferential diffusion on the flame extinction behaviors are similar to that of propane/air mixture, and the results in Figures 6 and 7 show that

Figures 8 and 9 are the results of the measured flame temperature profiles at different flame stretch rates with the mixture equivalence ratios of 0.65 and 1.79 , respectively. The temperatures in the figures were the raw data from the direct thermocouple readings and without any radiant correction. For each curve, the ending points were the visual locations of the twin flames. As can be seen from the curves, the temperature still increased as gas passing the visual flame surface.

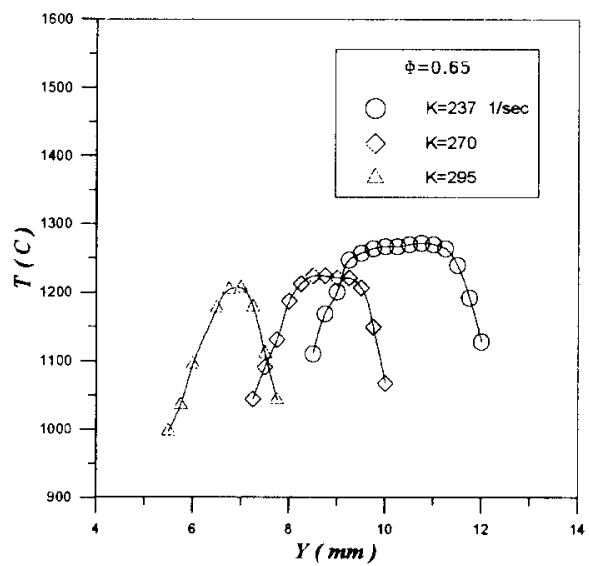

FIG. 8

The flame temperature distributions for mixture equivalence ratios of 0.65 at different stretch rates

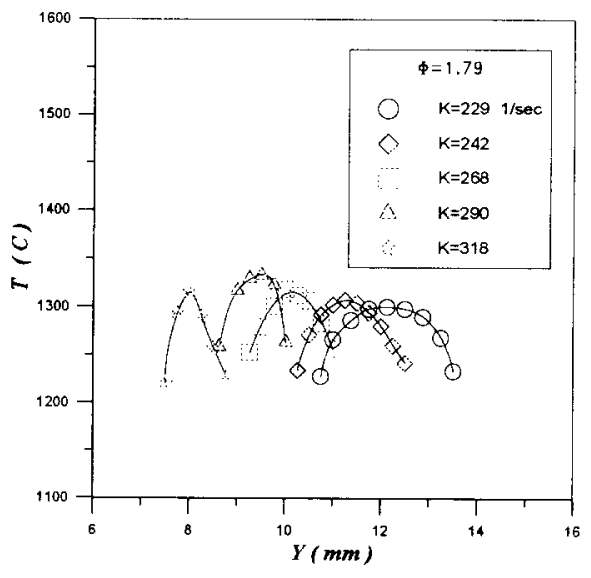

FIG. 9

The flame temperature distributions for mixture equivalence ratio of 1.79 at different stretch rates

Figure 10 is the plots of the flame and maximum temperature at different flame stretch rates with the equivalence ratio of 0.65 . As can be seen from the figure that both the flame and maximum temperatures decreased as the flame stretch rates increased, till the flames approached to the extinction. Figure 11 is the results for the equivalence ratio of 1.79 . And the results showed that the flame and maximum temperature increased initially as the flame was away from the extinction limit, but they started to decrease with further increasing the flame stretch till the flame approached to the extinction. The results are pretty much similar to the results of Sato [16] 
in propane/air mixtures, which prove the important of the effects of non-unity Lewis number and preferential diffusion on the ethanol/air flame extinction. In the theoretical work by Law [4] also predicted and explained quite well of such phenomena when the flames toward to the extinction.

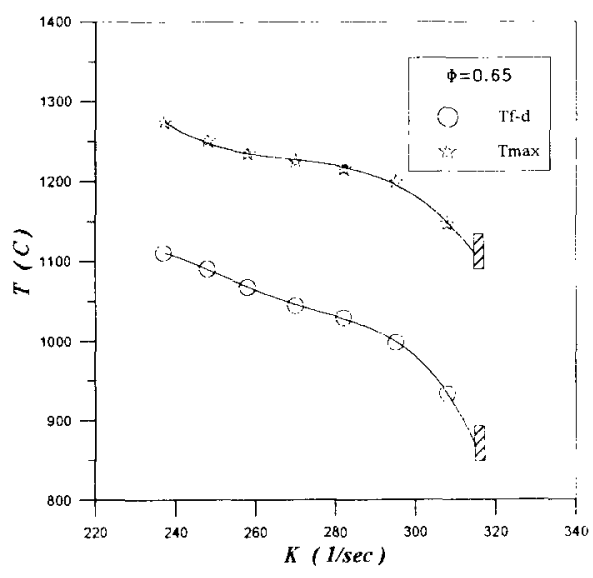

FIG. 10

The flame and the maximum temperatures vs. the flame stretch rates for mixture equivalence ratio of 0.65

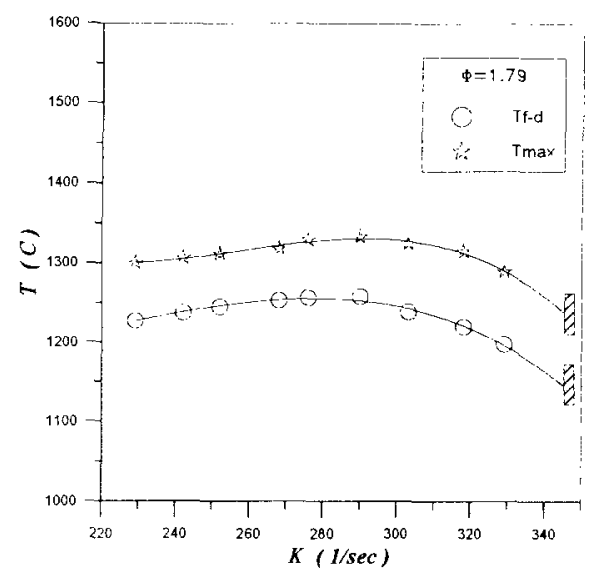

FIG. 11

The flame and the maximum temperatures vs. the flame stretch rates for mixture equivalence ratio of 1.79

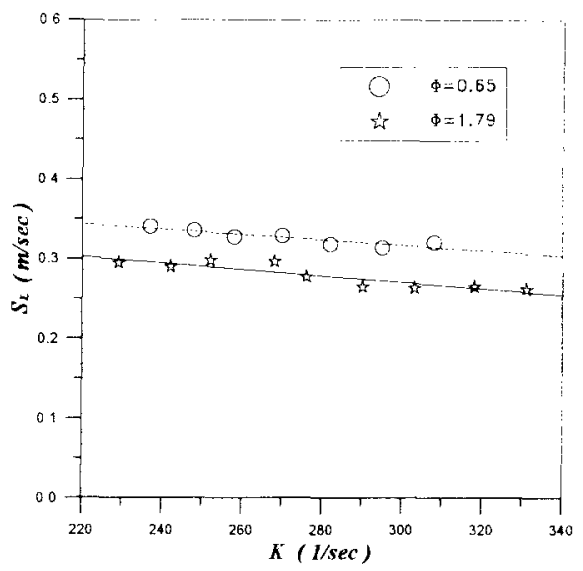

FIG. 12

The flame propagation velocities vs. the flame stretch rates for mixture equivalence ratio of 0.65 and 1.79

Finally, figure 12 is the results of the flame propagation velocity $\mathrm{S}_{\mathrm{L}}$ versus the flame stretch rates at equivalence ratio of 0.65 and 1.79 , respectively, where $S_{L}$ was the minimum velocity in 
the measured velocity profile. As can be seen from the figure that within the experimental stretch rate ranges, the tendency of $S_{L}$ decreased as the flame stretch rates increased both in the very lean and rich situations. The tendency is correct at the high stretch rates, since the flame temperature decreases as the stretch rate increases. At the medium stretch rate, it seems that there is a controversy in the equivalence ratio of 1.79 , since the flame temperature rising should increase the flame propagation velocity. However, if one looks through the data points more carefully, they did have such a tendency, that the flame propagation velocity increased as the stretch rate increased in the medium stretch rates region. A very important point might be concluded - when performing a linear extrapolation processes to determine the laminar flame propagation velocity and the extinction flame limits at zero stretch rate, the data points should not be adopted if the two flames are already merged, since the reaction mechanisms are already deviated from that the mixture should be possessed

\section{$\underline{\text { Conclusion }}$}

The only assumption imposed on determination of the flammability limits is the linear extrapolating of measured extinction limits to a zero stretch condition. However, due to the experimental difficulty of those pre-vaporized liquid ethanol/air mixture, the flame stretch rate was not sufficiently low. Thus, the extrapolating procedure might yield some errors. Further improving of the system to reduce the lower flame stretch rate may be necessary to yield more comfortable results.

The experimental results of the extinction limits at different flame stretch rates and mixture's preheated temperatures obtained herein are still valuable, and can be applied to validate the theoretic modeling of reaction and/or extinction mechanisms of fuels. In practice, the information presented herein can also help reduce the pollutant emissions from turbulent flame or engine combustion by preventing the flame extinguished from stretch due to flame curvature.

The effects of flame stretch and preheated mixture temperature on the flame extinction limits were accounted for adequately from Damk öler number point of view, and the flame extinction behaviors was described comfortable from the point of non-unity Lewis number effect or preferential diffusion effect of the mixture as they suffered a stretch.

Flame positions and the temperature variations at different flame stretch rates of lean and rich mixtures clear show the effects of non-unity Lewis number and preferential diffusion, which should be considered carefully, particularly as the flame close to the limits. 
The results of the flame propagation velocity further point out that the linear extrapolation process is not adequate if the flames close to the extinction limits, especially the two flames are merged.

\section{$\underline{\text { References }}$}

1. J. Warnatz, 18th Symposium (Int) on Combustion, 369 (1981).

2. C.K. Westbrook and F.L. Dryer, 18th Symposium (Int) on Combustion, 749 (1981)

3. C.K. Law and C.K. Wu, 20th Symposium (Int) on Combustion, 1941 (1984).

4. C.K. Law, 22nd Symposium (Int) on Combustion, 1381 (1989).

5. G. Dixon-Lewis, 23rd Symposium (Int) on Combustion, 305 (1990)

6. J.H. Tien and M. Matalon, Comb. \& Flame, vol. 84, 238 (1991).

7. C.M. Vagelopoulos, F.N. Egolfopoulos and C.K. Law, 25th Symposium (Int) on Combustion, 1341 (1994).

8. D.R. Dowdy, D.B. Smith, S.C. Taylor and A. Williams, 23rd Symposium (Int) on Combustion, 325 (1990).

9. D.P. Mishra, P.J. Paul and H.S. Mukunda, Comb. \& Flame, vol. 99, 379 (1994).

10. L.K. Tseng, M.A. Ismail and G.M. Faeth, Comb. \& Flame, vol. 95, 410 (1993).

11. C.J. Sung, C.K. Law and R.L. Axelbaum, Comb. Sci. and Tech, vol. 99, 119 (1994)

12. F.A. Williams, Combustion Theory, Combustion Sci. and Eng. Series (1985).

13. K.K Kuo, Principles of Combustion, John Wiley \& Sons (1986).

14. C.K. Law, D.L. Zhu and G. Yu, 21st Symposium (Int) on Combustion, 1419 (1986)

15. I. Glassman, Combustion, Academic Press Inc. (1987).

16. J. Sato, 19th Symposium (Int) on Combustion, 1541 (1982).

17. R.J. Kee, G. Dixon-Lewis, J. Warnatz, M.E. Coltrin and J.A. Miller, Sandia Report, SAND86-8246 (1993). 